\title{
MEMS Skin Friction Sensor with High Response Frequency and Large Measurement Range
}

\author{
Huihui Guo ${ }^{1,2}$, Xiong Wang ${ }^{3, *}$, Tingting Liu ${ }^{1, *}$, Zhijiang Guo ${ }^{1}$ and Yang Gao ${ }^{1}(\mathbb{D}$ \\ 1 School of Information Engineering, Southwest University of Science and Technology, Mianyang 621010, China; \\ easonguo@swust.edu.cn (H.G.); guozhijiang@swust.edu.cn (Z.G.); gaoy@swust.edu.cn (Y.G.) \\ 2 Robot Technology Used for Special Environment Key Laboratory of Sichuan Province, \\ Mianyang 621010, China \\ 3 Hypervelocity Aerodynamics Institute, China Aerodynamics Research and Development Center, \\ Mianyang 621010, China \\ * Correspondence: wangx81@cardc.cn (X.W.); liutingting@swust.edu.cn (T.L.); Tel.: +86-816-2465206 (X.W.); \\ $+86-816-6089322$ (T.L.)
}

check for updates

Citation: Guo, H.; Wang, X.; Liu, T.; Guo, Z.; Gao, Y. MEMS Skin Friction Sensor with High Response

Frequency and Large Measurement Range. Micromachines 2022, 13, 234. https://doi.org/10.3390/mi13020234 Academic Editors: Weidong Wang and Ruiguo Yang

Received: 29 December 2021

Accepted: 26 January 2022

Published: 30 January 2022

Publisher's Note: MDPI stays neutral with regard to jurisdictional claims in published maps and institutional affiliations.

Copyright: (C) 2022 by the authors. Licensee MDPI, Basel, Switzerland. This article is an open access article distributed under the terms and conditions of the Creative Commons Attribution (CC BY) license (https:// creativecommons.org/licenses/by/ $4.0 /)$.

\begin{abstract}
Micro-electromechanical system (MEMS) skin friction sensors are considered to be promising sensors in hypersonic wind tunnel experiments owing to their miniature size, high sensitivity, and stability. Aiming at the problem of short test duration (a few milliseconds) and heavy load in a shock wind tunnel, the fast readout circuit and the sensor head structures of a MEMS skin friction sensor are presented and optimized in this work. The sensor was fabricated using various micro-mechanical processes and micro-assembly technology based on visual alignment. Meanwhile, the sensor head structure was integrated with the fast readout circuit and tested by using a centrifugal force equivalent method. The calibration results show that this sensor provides good linearity, sensitivity, and stability. The measurement ranges are 0-2000 $\mathrm{Pa}$ with good performance. The resolution is better than $10 \mathrm{~Pa}$ at $3000 \mathrm{~Hz}$ detection frequency of the readout circuit for the sensor in ranges from 0 to $1000 \mathrm{~Pa}$. In addition, the repeatability and linearity of static calibration for sensors are better than $1 \%$.
\end{abstract}

Keywords: skin friction sensors; shock tunnel experiment; MEMS; fast readout circuit

\section{Introduction}

The skin friction resistance of an aircraft surface is an important part of its total resistance, which greatly limits the performance of hypersonic vehicles. Therefore, the skin friction measurement of an aircraft model is an important basic item in aerodynamic research. MEMS skin friction sensors are considered promising sensors in hypersonic wind tunnel experiments owing to their miniature size, high sensitivity, and stability. In recent years, several researchers have developed MEMS sensors to measure skin friction, including the capacitance-type and comb differential capacitance-type [1-5], piezoresistive-type [6-8], and piezoelectric-type [9]. For example, Mills et al. [5] reported a fully differential capacitive wall shear stress sensor for low-speed wind tunnels with the high sensitivity of $196 \mathrm{mV} / \mathrm{Pa}$ and a minimum detection limit of $12 \mathrm{mPa}$ at $1000 \mathrm{~Hz}$ in a range from 0-10 Pa; Von, P. et al. [8] reported a wall shear stress sensor using four piezoresistors in the cantilever, and the resolution was $0.01 \mathrm{~Pa}$ in the range of $2 \mathrm{~Pa}$; Kim, T. et al. [9] reported a piezoelectric floating element shear stress sensor for the wind tunnel flow measurement with the high sensitivity of $56.5 \mathrm{pc} / \mathrm{Pa}$ and with the frequency range of the sensor up to $800 \mathrm{~Hz}$. The common characteristics of those sensors are their usually high sensitivity, high resolution $\left(10^{-2} \mathrm{~Pa}\right)$, and small measurement range (several $\mathrm{Pa}$ ). Therefore, these sensors are mainly used in low wind tunnels to measure skin friction because their sensing elements, such as the comb capacitance, were exposed in the flow field. To adapt to the harsh measurement environments and large normal loads in hypersonic fields, a novel MEMS skin sensor was developed in our previous work $[10,11]$. The sensor adopts the floating element so that 
it is even with the measured wall, and the signal output micro-structure is isolated from the hypersonic field, which was fabricated using various micro-mechanical processes and micro-assembly technology. Experimental results show that this sensor has some good characteristics, such as good linearity, small size, high sensitivity, and repeatability of static calibration. Furthermore, the measurement ranges were 0-100 $\mathrm{Pa}$, and the minimum detectable skin friction was 0.1 Pa. However, the natural frequency of the sensor was $410 \mathrm{~Hz}$, and the detection frequency of a weak capacitance readout circuit for a skin friction sensor is only $10 \mathrm{~Hz}$.

According to the hypersonic flow field characteristics of high temperature, high total pressure, and short test time in the shock wind tunnel, the test ambient temperature is usually increased to hundreds of degrees centigrade, and the measurement ranges increase to hundreds, or even thousands, of Pa. Meanwhile, the sampling frequency of the readout circuit for the sensor also increases to thousands of $\mathrm{Hz}$ with higher loads, and only several milliseconds in duration of the working time. Therefore, greater measurement ranges and higher response frequencies of sensors for skin friction in shock wind tunnels need to be developed.

In this work, the static force analysis and modal analysis of the sensor head structure were carried out by finite element analysis (FEA) to optimize the head structure of the MEMS skin friction sensor with a high response frequency and large measurement ranges. In addition, to improve the detection frequency of the weak capacitance of the sensor, the fast readout circuit with $3000 \mathrm{~Hz}$ detection frequency was designed and integrated with the sensor head structure.

\section{Structural Simulation and Optimization of the Sensor Head Structure}

The structure of the MEMS skin friction sensor is shown in Figure 1. The sensor was composed of a silicon differential capacitor embedded with a floating element, a signal readout circuit, and a metal shell package. The working principle of this sensor is explained as the skin friction caused the torsional deflection of clamped-clamped elastic beams by the floating element, and the torsional deflection was transferred into a capacitance variation by one pair of differential capacitors.

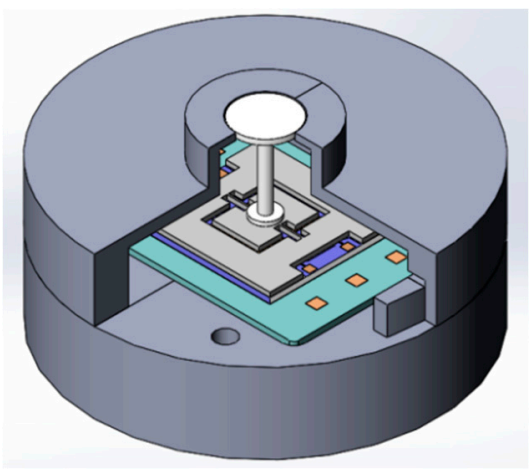

(a)

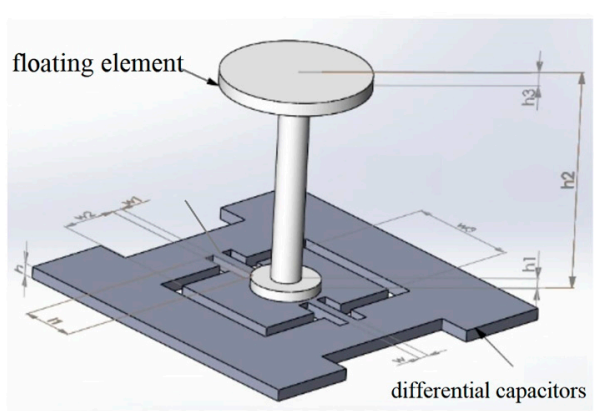

(b)

Figure 1. (a) Schematic of the sensor; (b) the sensing structure parameters of the sensor head.

For a typical shock tunnel with a short test time (a few milliseconds), the lowest frequency to obtain high measurement accuracy within the effective test time is $1000 \mathrm{~Hz}$. Because the mechanical vibration of the measurement system is excited and cannot be attenuated rapidly enough by damping characteristics during the short test time, to obtain higher measurement accuracy, the natural frequency of the MEMS skin friction sensor should, therefore, be raised to $3000 \mathrm{~Hz}$. 
For an undamped single freedom system, the natural frequency calculation formula is defined as shown in the following equation:

$$
f_{n}=\frac{1}{2 \pi} \sqrt{\frac{k}{m}}
$$

where $f_{n}$ is the natural frequency, and $k$ and $m$ are the stiffness and mass of the structure, respectively. From Equation (1), it can be seen that increasing the stiffness of the beam and reducing the mass of the floating element was an effective way to improve the natural frequency of a MEMS skin friction sensor. Then, the sensitivity of the sensor deteriorated rapidly because the torsional deflection of the elastic beams decreased with an increase in the stiffness (with increasing $w$ and $h$ ) under the same loads. The sensitivity of the sensor can be defined according to conformal transformation theory [11]:

$$
S \approx \frac{\tau_{w} A}{\Delta C}=\frac{h_{0}^{2}}{\varepsilon_{0}\left(2 w_{1}+w_{2}\right) w_{2} w_{3}} \times \frac{\beta}{1+v} \times \frac{E w^{3} h}{l_{1} h_{2}}
$$

where $\tau_{w}$ is the measured skin friction, $\Delta C$ is the variation of differential capacitance, $\mathrm{A}$ is the area of the floating element, $\beta, v, \varepsilon_{0}$ and $E$ are the elastic deformation parameters of the torsion beam, respectively, $w, l_{1}$ and $h$ are the length width and thickness of the silicon beam, and $h_{0}$ and $h_{2}$ are the gaps of the capacitor and the height of the floating element as a force arm. To fabricate the high-performance skin friction sensor for a shock wind tunnel experiment, the sensitivity and the natural frequency were considered together.

To obtain the main structural parameters of the silicon beam and the floating element, three-dimensional FEA models of the sensor head structures were built with the help of FEA software, using our previous parameters. The optimized three-dimensional FEA model of the sensor head structure is shown in Figure 2a.

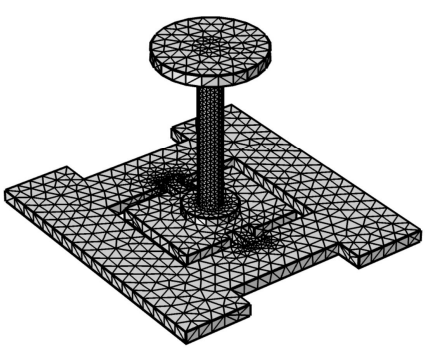

(a)

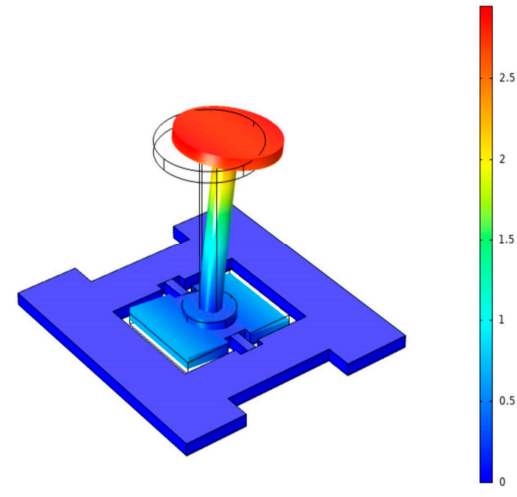

(b)

Figure 2. (a) Three-dimensional FEA model of the sensor head structure; (b) the normal deformation of the vibrating plate under 2000 Pa equivalent loads.

Combining the designed indexes and our previous research results, we determined that increasing the width of the silicon beam was key to improving the stiffness of the sensor head structure. To ensure the working state of the torsion beam, the width was not allowed to exceed its thickness (the thickness of the wafer was about $500 \mu \mathrm{m}$ ). The natural frequency of the sensor head with different widths of the silicon beam is shown in Figure 3a. It can be seen that the natural frequency of the sensor head was from $410 \mathrm{~Hz}$ to $1000 \mathrm{~Hz}$ as the width of the silicon beam was increased from $180 \mu \mathrm{m}$ to $420 \mu \mathrm{m}$. However, the natural frequency increase tended to slow down when the width of the silicon beam was increased to $420 \mu \mathrm{m}$. Meanwhile, the sensitivity of the sensor was down 90 percent by Equation (2). The maximum width of the beam can be determined as $420 \mu \mathrm{m}$ without considering the sensitivity of the sensor in this work. 


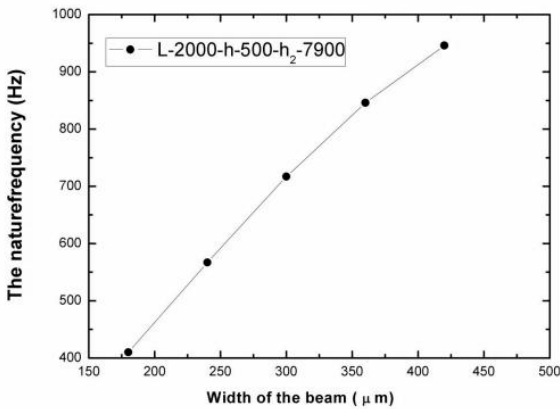

(a)

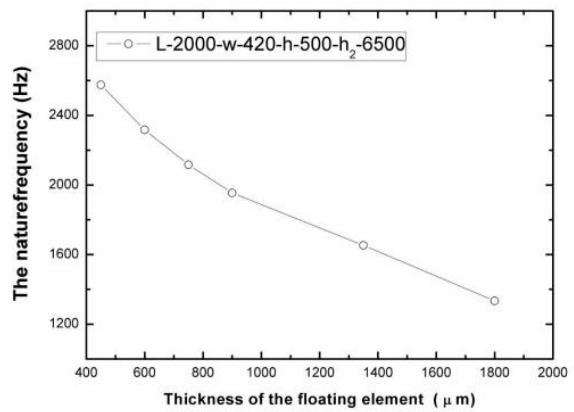

(c)

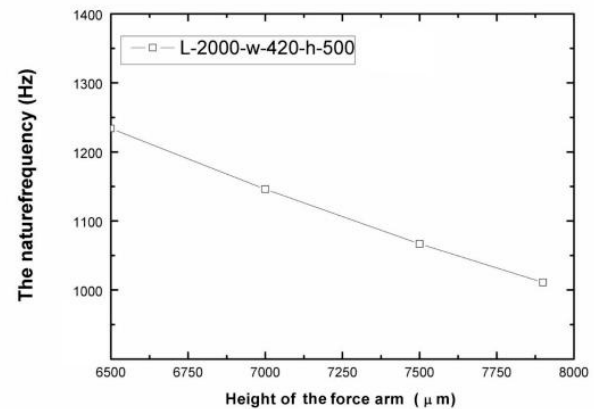

(b)

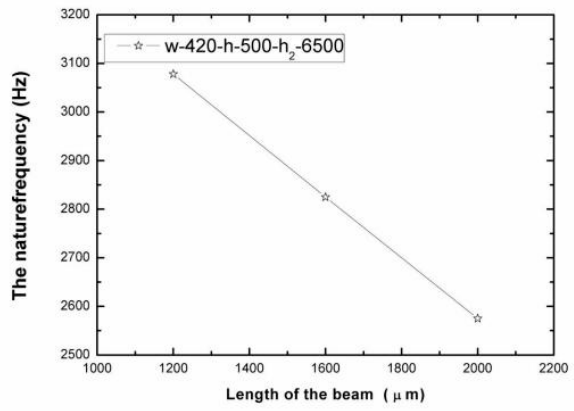

(d)

Figure 3. The variation of the natural frequency with different structure parameters of the sensor head: (a) the width changes of the silicon beam; (b) the height changes of the force arm; (c) the thickness changes of the floating element; (d) the length changes to the silicon beams.

To further enhance the natural frequency, the height of the floating element as a force arm and the mass of the floating element were reduced. The frequency increased from $1000 \mathrm{~Hz}$ to $1250 \mathrm{~Hz}$ as the height of the force arm was decreased from $7900 \mu \mathrm{m}$ to $6500 \mu \mathrm{m}$, as shown in Figure 3b. It can also be seen that reducing the force arm had little effect on improving the natural frequency of the sensor head. The reason is that the reduction of the length of the floating element has little effect on the overall quality of $\mathrm{m}$.

Therefore, reducing the thickness of the floating element can quickly reduce its overall mass to improve the natural frequency of the sensor, as shown in Figure 3c. However, the rapid reduction of the overall mass led to the rapid reduction of the equivalent torque and the sensitivity. Combining the influence rule of the skin friction measurements model $[12,13]$ and the precision machining process, the thickness parameters of the floating element were determined as $450 \mu \mathrm{m}$ in this work.

The influence of the length of the beam on the natural frequency of the sensor was also simulated, as shown in Figure 3d. The simulation results show that the natural frequency of the sensor head increased linearly with the decreasing length of the beam.

Finally, to increase the natural frequency of the sensor to over $3000 \mathrm{~Hz}$, the main structure parameters were achieved, as shown in Table 1.

Table 1. The main structural parameters of the sensor.

\begin{tabular}{|c|c|c|c|c|c|c|}
\hline $\begin{array}{c}\text { Width of Silicon } \\
\text { Beam } \\
(\mu \mathrm{m})\end{array}$ & $\begin{array}{c}\text { Length of Silicon } \\
\text { Beam } \\
(\mu \mathrm{m})\end{array}$ & $\begin{array}{l}\text { The Thickness of } \\
\text { the Silicon Beam } \\
(\mu \mathrm{m})\end{array}$ & $\begin{array}{l}\text { Diameter of the } \\
\text { Floating Element } \\
(\mu \mathrm{m})\end{array}$ & $\begin{array}{c}\text { Thickness of } \\
\text { Floating Element } \\
(\mu \mathrm{m})\end{array}$ & $\begin{array}{c}\text { Height of } \\
\text { Floating Element } \\
(\mu \mathrm{m})\end{array}$ & $\begin{array}{c}\text { Gap of Capacitor } \\
(\mu \mathrm{m})\end{array}$ \\
\hline 400 & 1200 & 500 & 5000 & 450 & 7000 & 10 \\
\hline
\end{tabular}

Meanwhile, the skin friction $\tau_{w}$ was equivalent to the shear stress load on the surface of the floating element in the finite element simulation. The displacement of the differential capacitor plate under $2000 \mathrm{~Pa}$ loads is shown in Figure 2b. It can be seen that the maximum normal deformation of the edge of the vibrating plate away from the torsion beam was 
$+1.06 \mu \mathrm{m}$ and $-1.05 \mu \mathrm{m}$, respectively. To ensure the good linearity of the sensor in the total measurement range, the gap of the differential capacitor was larger than $6 \mu \mathrm{m}$. In this work, the gap of the differential capacitor was designed as $10 \mu \mathrm{m}$, and the initial capacitance was about $9 \mathrm{pF}$ by calculation. At the same time, the design sensitivity was about $2.5 \mathrm{~Pa} / \mathrm{fF}$ in the range of 0-2000 Pa by Equation (2).

\section{The Fast Readout Circuit Design}

Considering the previous simulation and calculation results, the variation of the capacitance under the skin friction load was very small, within $0.01 \sim 1.00 \mathrm{pF}$. Thus, the circuit needs not only high measurement precision but also a high detection frequency for a shock tunnel with the short time of a few milliseconds. To improve the measurement precision, the signal wire between the sensor head and readout circuit were as short as possible, thus, the readout circuit was integrated with the MEMS chip, as shown in Figure $4 \mathrm{a}$. The relationship between the detection frequency and the measurement accuracy of the sensor was contradictory. It was key to select a weak capacitance detection chip with a high detection frequency and measurement accuracy. The readout circuit included weak capacitance testing, and the processing circuit part and the software real-time display part were designed in this work. A Pcap01 chip is an application-specific integrated circuit for a MEMS capacitance sensor with a low-power capacitance-to-digital converter with a wide input range ( $\mathrm{fF}$ to $\mathrm{nF}$ ) and versatile configuration options, and it can be configured for the highest sampling rates of up to $500 \mathrm{kHz}$, the lowest current consumption of down to $2 \mathrm{uA}$, or the lowest noise of $15 \mathrm{aF}$ (RMS). In this work, the capacitance signal testing and processing circuit were mainly composed of a Pcap01 chip and single chip (STM32F411CEU6) due to the limitation of the volume, as shown in Figure 4b, which was fabricated by a precision ceramic micro-strip circuit process. The real-time detection and display functional interfaces of the MEMS skin friction sensor were designed using LabVIEW software, as shown in Figure 4c.

Although the measurement accuracy deteriorated with the increase in sampling frequency, the readout circuit for the MEMS skin sensor still had sufficiently high detection accuracy at $3000 \mathrm{~Hz}$ to meet the testing requirements in a shock wind tunnel. The resolution was about $10 \mathrm{~Pa}$ by static calibration test using a centrifugal force equivalent method.

The components of the MEMS skin friction sensors, including the MEMS chip, floating element, signal readout circuit, and package shell, were fabricated using MEMS and precision machining process technology. MEMS chips were fabricated using the MEMS process, which consists of a silicon structure and glass electrode substrate. The photo of a single chip is shown in Figure 5a. The signal readout circuit was fabricated by precise microstrip circuit technology to ensure the precision of the components and processing. The photo of the ceramic circuit board is shown in Figure 5b. The material of the floating elements and package shells was aluminum alloy to be consistent with the test model material. The precision turning and milling technologies with $10 \mu \mathrm{m}$ geometrical precision were used to fabricate the floating element and package shells. A photo of the separate components is shown in Figure 5c.

The accuracy of the skin friction measurements is very closely related to the assembly error of the sensor and the flow characteristics of the measured wall [11]. To reduce assembly error, a micro-assembly method for MEMS skin friction sensor assembly based on visual alignment was developed in our previous work [10]. The key parts mainly included the floating element and MEMS chip assembly, the MEMS chip and readout circuit assembly, and the sensor head and package assembly, which are shown in Figure 5d. 


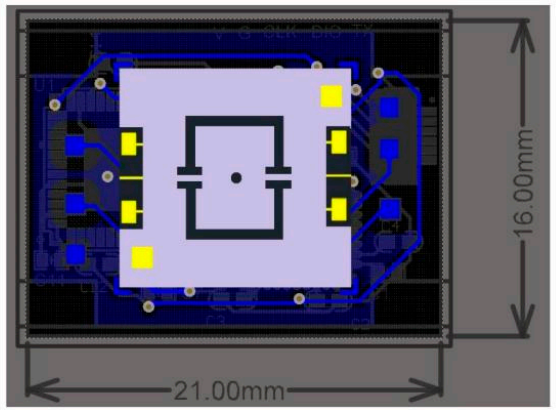

(a)

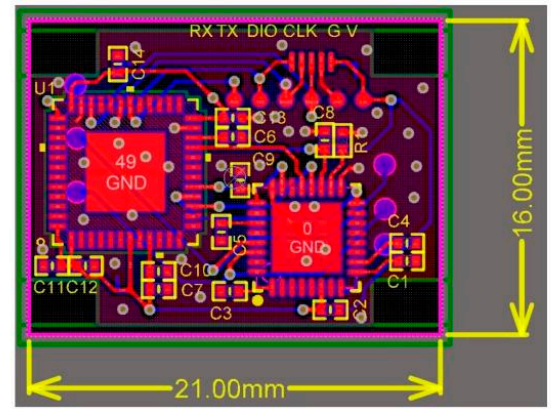

(b)

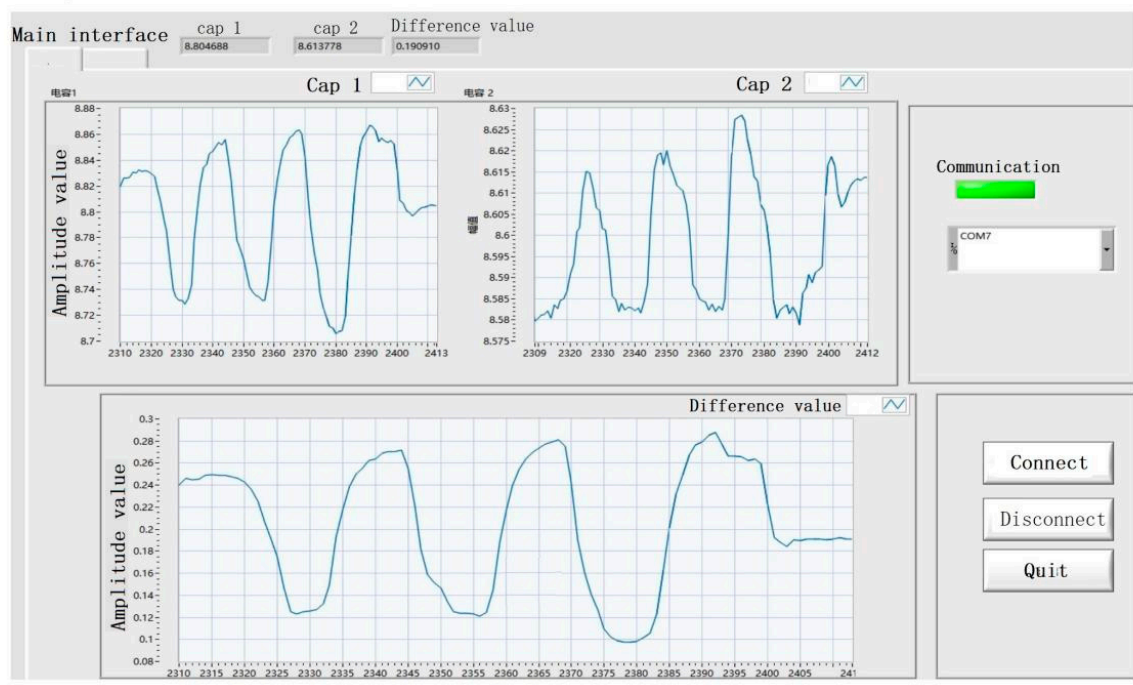

(c)

Figure 4. (a) Schematic of the readout circuit integrated with MEMS chip; (b) the PCB of the circuit; (c) the display functional interfaces for the MEMS skin friction sensor.

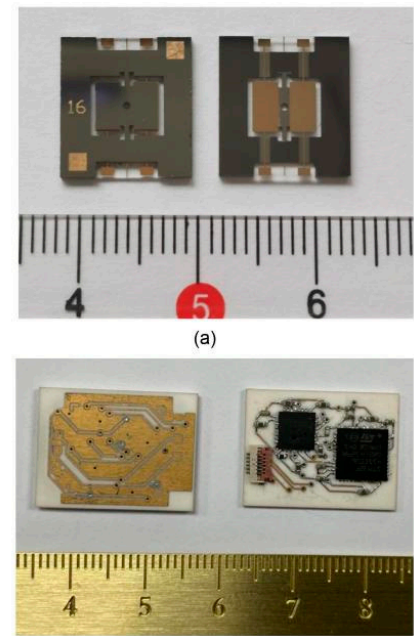

(b)

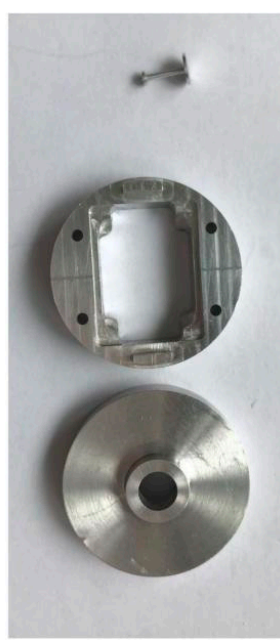

(c)

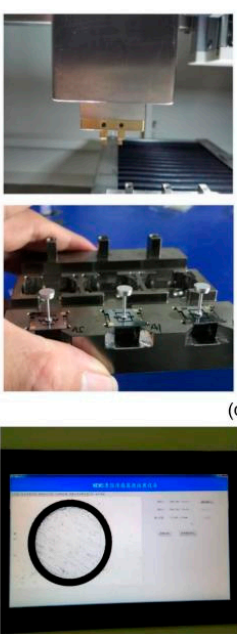

(e)
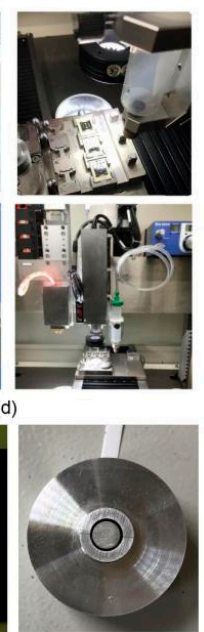

(f)

Figure 5. The photos of the separate components for MEMS sensor $(\mathbf{a}-\mathbf{c})$; (d) the micro-assembly processes for MEMS sensor; (e) the coaxiality measurement for sensor; (f) the photo of the assembled sensor.

The assembly results show that the assembly error can be well controlled by using visual alignment and a micro-operating assembly system. The coaxiality of the MEMS skin friction sensor was very good, and the error was only $2 \mu \mathrm{m}$ by calculating pixels, as shown 
in Figure 5e. A photo of the sensor with good electrical characteristics and small assembly error is shown in Figure $5 \mathrm{f}$.

\section{Static Calibration of the Sensor}

The performances of the MEMS skin friction sensor were determined after assembly. With an experiment of static loading force, the static performance parameters of the sensor were obtained. Due to the small skin friction and volume of the MEMS sensor, the centrifugal force equivalent method and a single-spindle rotary loading platform were adopted to statically calibrate the sensor [11]. The static calibration system for the sensor is shown in Figure 6.

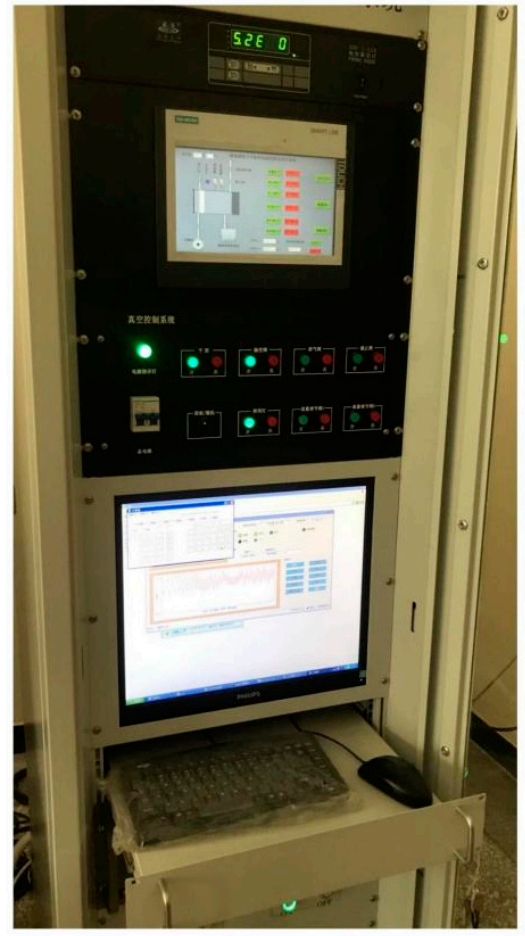

(a)

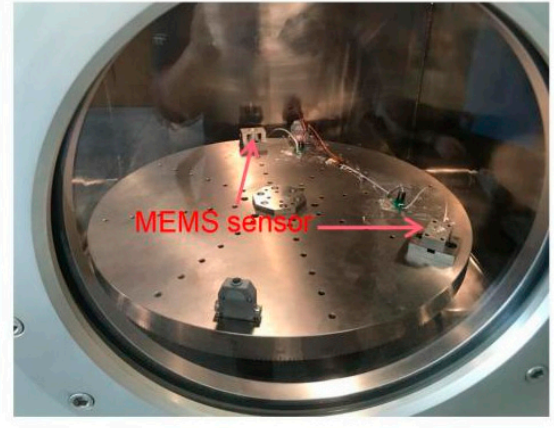

(b)

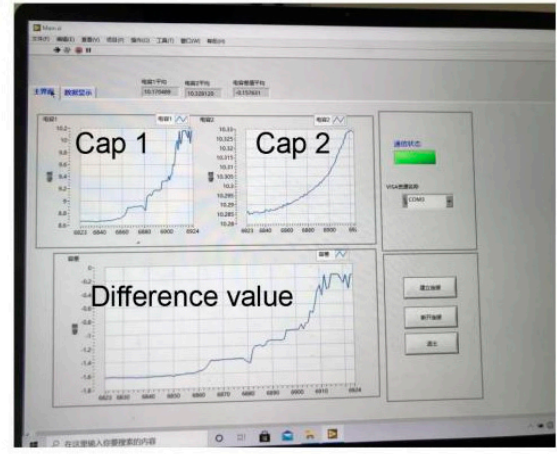

(c)

Figure 6. (a) Control system of the loading platform; (b) rotary loading platform in vacuum environment; (c) signal real-time detection, storage, and display window of the MEMS skin friction sensor.

The MEMS sensors were fixed onto a turntable in a vacuum environment, as shown in Figure $6 \mathrm{~b}$. The skin friction sensed by the floating element was in some cases equivalent to the rotating speed of the turntable when the structure parameters of the sensor were determined. Then, the relationship between the equivalent friction and capacitance were obtained. The variation of capacitance under the equivalent friction loads was displayed and stored in real time, as shown in Figure 6c.

The maximum equivalent load was set to $1000 \mathrm{~Pa}$, which was limited by the rotating speed of the turntable. The output response of the MEMS skin friction sensor under different equivalent loads is shown in Figure 7. The detection limit of the sensors was determined by the head structure of the sensor and the noise of the readout circuit. The inset shows the responses of the sensor under small equivalent loads. It can be seen that the response curve displays a clear stepwise increase when the load was increased from 0 to $10 \mathrm{~Pa}$. The resolution of the sensor was better than $10 \mathrm{~Pa}$ at the same time. 
(b)

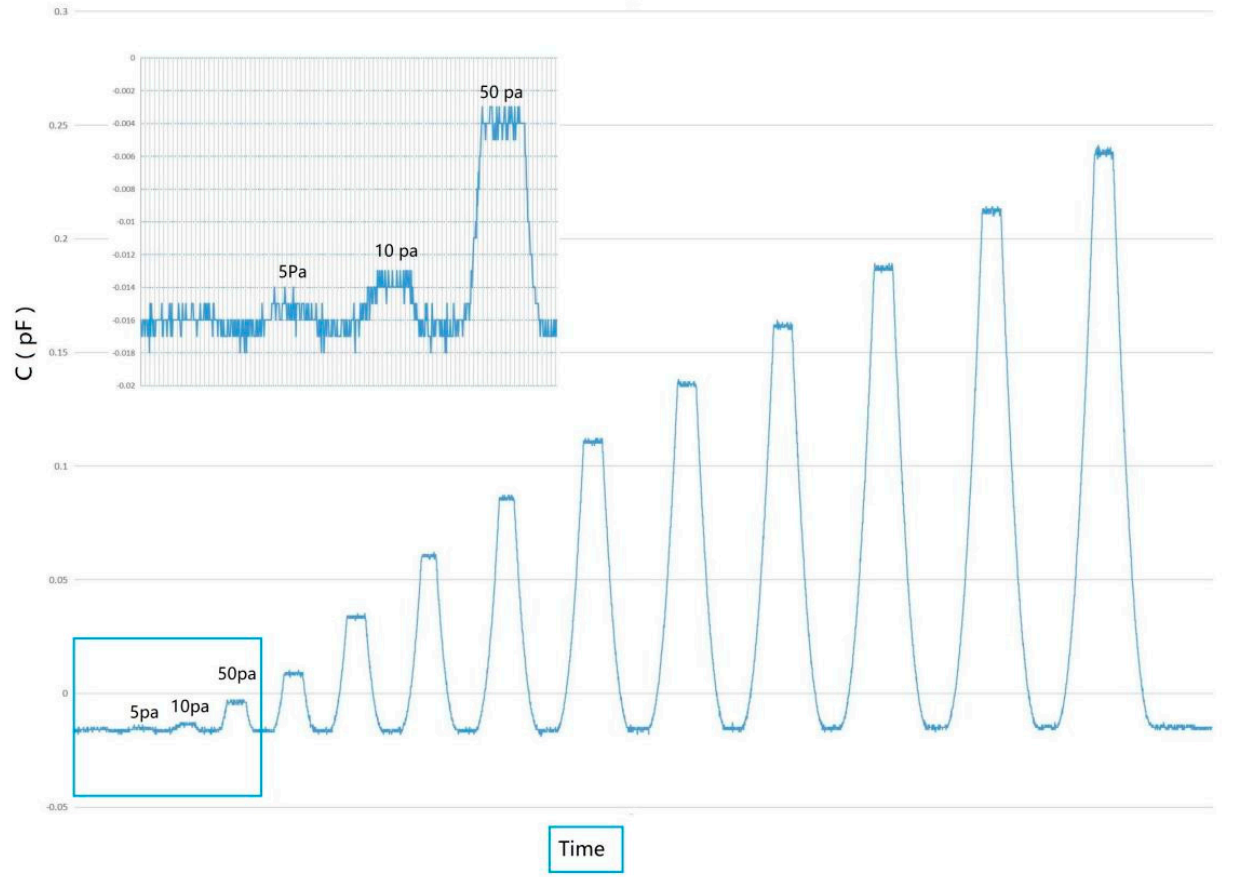

Figure 7. (a) The output response of the MEMS skin friction sensor under different equivalent loads; (b) the output response of the sensor under small loads.

To obtain the repeatability characteristics of the sensor, seven groups of response values under the same loading conditions were collected and stored at one time. To obtain the response curve of the sensor under different loads, the response values were averaged during a steady load. The repeatability curve of sensor \#1 under different loads is shown in Figure 8a. It can be seen that the sensor has good linearity and stability. The linearity and repeatability are both better than $1 \%$ in the range from 0 to $1000 \mathrm{~Pa}$, the sensitivity is about 3.8 Pa/fF, and the detection limit is about $10 \mathrm{~Pa}$. In addition, the inset of Figure 8a shows the responses of the sensor in a range up to $100 \mathrm{~Pa}$.

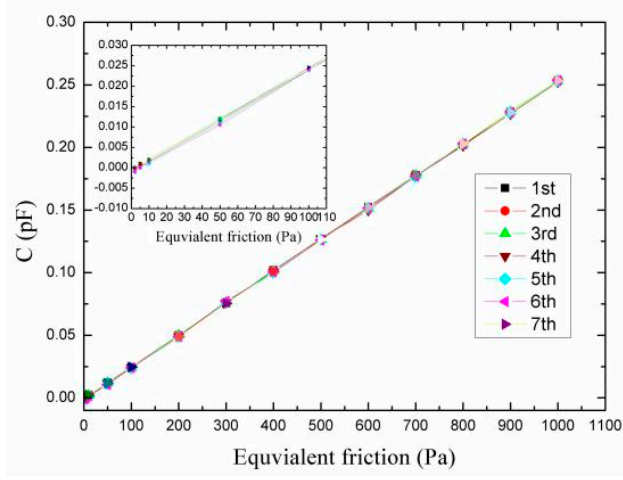

(a)

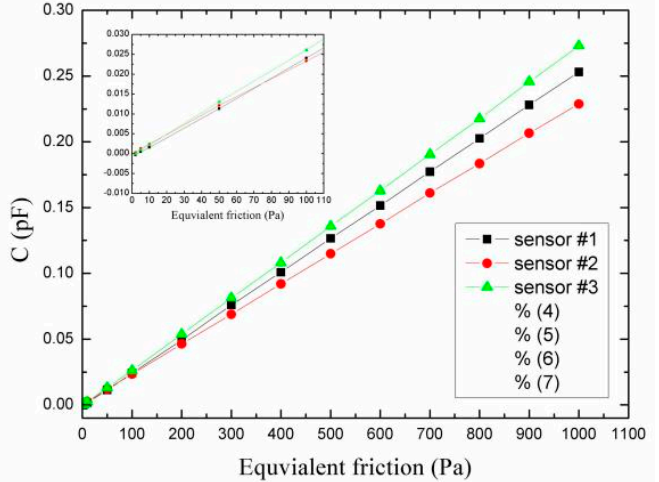

(b)

Figure 8. (a) The repeatability curves of sensor \#1; (b) the response curves of three sensors under different loads.

Although all components of the sensors were manufactured in the same batch, the machining errors and assembly errors still affected the consistency of the sensors. The response characteristics of the three sensors are shown in Figure 8b. Those sensors also had good linearity and stability in the range of 0 to $1000 \mathrm{~Pa}$. The sensitivities were $3.8 \mathrm{~Pa} / \mathrm{fF}$, $4.3 \mathrm{~Pa} / \mathrm{fF}$, and 3.6 Pa/fF, respectively, and the resolution was better than $10 \mathrm{~Pa}$, which met the needs of the shock wind tunnel experiment. 


\section{Conclusions}

This paper developed a MEMS skin friction sensor with high detection frequency and a large measurement range in a shock wind tunnel experiment. With the help of finite element analysis software, the sensor head structure of an MEMS sensor was optimized to enhance the natural frequency to $3000 \mathrm{~Hz}$. A key factor was to quickly increase the natural frequency of the sensor head with coarse-tuning by increasing the width of the beam and reducing the mass of the floating element. Then, the length of the torsional beam and the floating element as the force arm was used to achieve fine-tuning. However, the sensitivity deteriorated rapidly with the increasing natural frequency. In addition, to meet the testing requirement in the shock wind tunnel with a short test duration (a few milliseconds), it was key to design a weak capacitance detection circuit with high detection frequency and measurement accuracy. The readout circuit of the sensor was designed and integrated with the sensor head. The components of the sensor, including the MEMS chip, floating element, signal readout circuit, and package shell, were fabricated by using various micro-mechanical processes.

The sensors were assembled using micro-assembly technology based on visual alignment with small assembly errors. The coaxiality of the MEMS skin friction sensor was very good, and the error was only $2 \mu \mathrm{m}$. This is the main reason for the good manufacturing consistency of the sensor.

Then, the static performance parameters of the sensor were obtained using the centrifugal force equivalent method and the single-spindle rotary loading platform. The calibration results show that the sensors have good linearity and stability in a range from 0 to $1000 \mathrm{~Pa}$. The repeatability and linearity of static calibration for sensors are better than $1 \%$, and the resolution is better than $10 \mathrm{~Pa}$ at the $3000 \mathrm{~Hz}$ detection frequency of the sensor. Due to the maximum deformation values of the vibrating plate under 2000 Pa being only 10 percent of the differential capacitor gap, the sensor will have good linearity in a large range (greater than $2000 \mathrm{~Pa}$ ). Moreover, the gap of the differential capacitor is also one of the important parameters affecting the sensitivity of the sensor. The reduction of the capacitance gap is a key factor to quickly enhance the sensitivity of the sensor. For example, the sensitivity will double with the decrease of the gap of the capacitor from $10 \mu \mathrm{m}$ to $7 \mu \mathrm{m}$ in future work.

Author Contributions: Conceptualization, X.W. and H.G.; methodology, T.L.; Validation, H.G. and Y.G., data curation, Z.G.; writing — original draft preparation, H.G.; writing-review and editing, X.W.; visualization, Z.G.; project administration, H.G.; funding acquisition, H.G. All authors have read and agreed to the published version of the manuscript.

Funding: This research received no external funding.

Institutional Review Board Statement: Not applicable.

Informed Consent Statement: Not applicable.

Data Availability Statement: Data are contained within the article.

Conflicts of Interest: The authors declare no conflict of interest.

\section{References}

1. Jiang, Z.; Farmer, K.R.; Vijay, M. A MEMS device for measurement of skin friction with capacitive sensing. In Proceedings of the Microelectromechanical Systems Conference, Interlaken, Switzerland, 21-25 January 2001; Volume 8, pp. 4-7.

2. Zhao, Z.; Gallman, J.; White, R. A MEMS floating element with bump shear stress sensor array on a chip. In Proceedings of the 51st AIAA Aerospace Sciences Meeting Including the New Horizons Forum and Aerospace Exposition, Grapevine, TX, USA, 7-10 January 2013; p. 626.

3. Meloy, J.; Griffin, J.; Sells, J.; Cattafesta, L.; Sheplak, M.; Chandrasekharan, V. Experimental verification of a MEMS-based skin friction sensor for quantitative wall shear stress measurement. In Proceedings of the 41st AIAA Fluid Dynamics Conference and Exhibit, Honolulu, HI, USA, 27-30 June 2011; Volume 6, pp. 1-18.

4. Freitas, B.; Mills, D.A.; Keane, C.; Kelley, L.S.; Sheplak, M. Development of a two-dimensional wall shear stress sensor for wind tunnel applications. In Proceedings of the AIAA SciTech, San Diego, CA, USA, 5-9 January 2019; p. 2045. 
5. Mills, D.A.; Patterson, W.C.; Keane, C.; Sheplak, M. Characterization of a fully-differential capacitive wall shear stress sensor for low-speed wind tunnels. In Proceedings of the 2018 AIAA Aerospace Sciences Meeting, Kissimmee, FL, USA, 8-12 January 2018; p. 301.

6. Brian, A.A.; Park, S.J.; Mukundan, V.; Pruitt, B.L. Design and characterization of microfabricated piezoresistive floating elementbased shear stress sensors. Sens. Actuators A Phys. 2007, 134, 77-87.

7. Weiss, J.; Jondeau, E.; Giani, A.; Charlot, B.; Combette, P. Static and dynamic calibration of a MEMS calorimetric shear-stress sensor. Sens. Actuators A Phys. 2017, 265, 211-216. [CrossRef]

8. Von Papen, T.; Buder, U.; Ngo, H.D.; Obermeier, E. A second generation MEMS surface fence sensor for high-resolution wall shear stress measurement. Sens. Actuators A Phys. 2004, 113, 151-155. [CrossRef]

9. Kim, T.; Saini, A.; Kim, J.; Gopalarathnam, A.; Zhu, Y.; Palmieri, F.L.; Jiang, X. Piezoelectric floating element shear stress sensor for the wind tunnel flow measurement. IEEE Trans. Ind. Electron. 2016, 64, 7304-7312. [CrossRef] [PubMed]

10. Wang, X.; Zhu, T.; Xu, X.; Shi, Y. Fabrication, calibration and proof experiments in the hypersonic wind tunnel for a novel MEMS skin friction sensor. Microsyst. Technol. 2017, 23, 3601-3611. [CrossRef]

11. Xiong, W.; Nantian, W.; Xiaobin, X.; Tao, Z. Fabrication and Hypersonic Wind Tunnel Validation of a MEMS Skin Friction Sensor Based on Visual Alignment Technology. Sensors 2019, 19, 3803. [CrossRef] [PubMed]

12. Meritt, R.J.; Dunbar, M.J.; Molinaro, J.N.; Schetz, J.A. Error source studies of direct measurement skin friction sensors. In Proceedings of the 53rd AIAA Aerospace Sciences Meeting, Kissimmee, FL, USA, 5-9 January 2015; p. 1916.

13. Tian, Y.; Han, Y.; Yang, S.; Zhong, F.; Le, J. Investigation of fluctuating characteristics of wall shear stress in supersonic flow. Phys. Fluids 2019, 31, 125110. 